\title{
AURORA KINASES: STRUCTURE, FUNCTIONS AND THEIR ASSOCIATION WITH CANCER
}

\author{
Madhu Kollareddy, Petr Dzubaka, Daniella Zheleva ${ }^{\mathrm{b}}$, Marian Hajduch ${ }^{\mathrm{a} *}$
}

\author{
a Laboratory of Experimental Medicine, Department of Pediatrics, Faculty of Medicine, Palacky University, Puskinova 6 , \\ 77520 Olomouc, Czech Republic \\ b Cyclacel Pharmaceuticals, Inc. Dundee Technopole, James Lindsay Place, Dundee, Scotland, UK, DDI 5JJ \\ e-mail: hajduchm@gmail.com
}

Received: November 30, 2007; Accepted: January 18, 2008

Key words: Aurora kinases/Serine/threonine/Spindle/Centrosomes/Cytoskeleton/Cytokinesis/Oncogenes

Background: Aurora kinases are a recently discovered family of kinases (A, B \& C) consisting of highly conserved serine $\mid$ threonine protein kinases found to be involved in multiple mitotic events: regulation of spindle assembly checkpoint pathway, function of centrosomes and cytoskeleton, and cytokinesis. Aberrant expression of Aurora kinases may lead to cancer. For this reason the Aurora kinases are potential targets in the treatment of cancer. In this review we discuss the biology of these kinases: structure, function, regulation and association with cancer.

Methods and Results: A literature search.

Conclusion: Many of the multiple functions of mitosis are mediated by the Aurora kinases. Their aberrant expression can lead to the deregulation of cell division and cancer. For this reason, the Aurora kinases are currently one of the most interesting targets for cancer therapy. Some Aurora kinase inhibitors in the clinic have proven effectively on a wide range of tumor types. The clinical data are very encouraging and promising for development of novel class of structurally different Aurora kinase inhibitors. Hopefully the Aurora kinases will be potentially useful in drug targeted cancer treatment.

\section{INTRODUCTION}

\section{Aurora Kinases and the Cell Cycle}

The Cell cycle is an ordered set of events, culminating in cell growth and division into two daughter cells. The segregation of genetic material into two exact halves is the hallmark of cell division. The chromosomal DNA is replicated during $\mathrm{S}$ phase (DNA synthesis). The G1 phase or first gap phase is the interval between mitosis and DNA replication. The G2 phase or second gap phase is the interval between completion of DNA replication and mitosis. Mitosis is divided into five distinct stages (prophase, prometaphase, metaphase, anaphase and telophase) and the end of mitosis, two daughter cells are formed from one parent cell by cytokinesis. In brief, prophase begins after the transition from $\mathrm{G} 2$ to mitosis and chromosome condensation and disassembly of the nuclear envelope are key events. Attachment of chromosome kinetochores to microtubules and the assembly of chromosomes at the center of the cell to form the metaphase plate are the main events in prometaphase and metaphase respectively. Sister chromatids move to opposite pole and the poles move apart during anaphase. During telophase, the chromosomes recondense and the nuclear envelop reforms around genetic material. The most dramatic change in cellular structure at this time is constriction of the cleavage furrow and subsequent cytokinesis (Fig. 1).

These strict molecular events taking place in strict order during the cell cycle is responsible for reliable cell division to produce two daughter cells as precise copies of the parent cell. Biochemical points termed checkpoints, control transitions in the cell cycle to ensure the fidelity and progression into following stage. Mutations or over expression of the genes involved in these checkpoints can cause cancer. Such genes are called oncogenes. The first checkpoint occurs at the end of G1 phase and before $S$ phase, called G1 checkpoint or restriction checkpoint. The second checkpoint occurring at the end of G2 phase and before mitotic phase is called the G2 checkpoint. Apart from these two checkpoints one more stage called

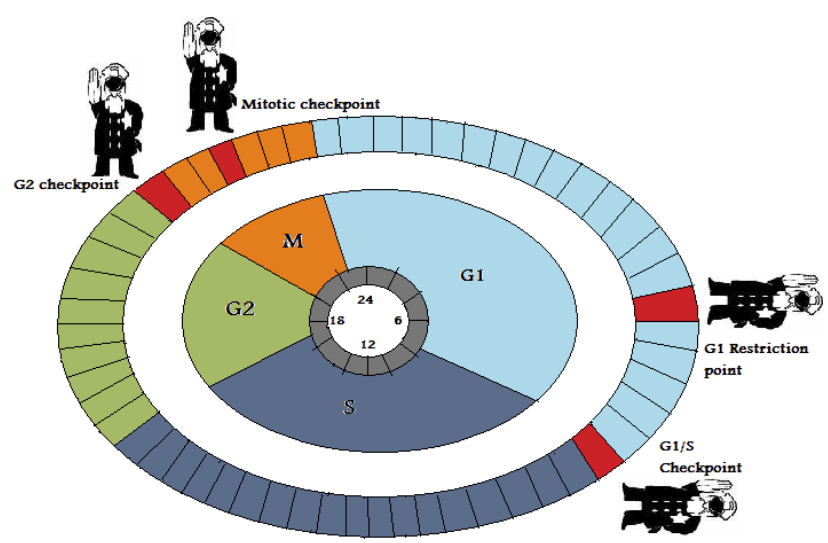

Fig. 1. Schematic cell cycle diagram showing phases of cell cycle and checkpoints. G1, S, G2, \& M phase are represented by different colors. Red boxes represent the checkpoints at each phase of the cell cycle. At each checkpoint stop signal exist represented by policeman, to prevent uncontrolled cell division. 
anaphase checkpoint occur during metaphase and checks for proper alignment of metaphase chromosomes, which should be in a state of bipolar tension. Checkpoints involve orderly synthesis, activation, degradation, phosphorylation and dephosphorylation of different proteins involved in cell cycle (Fig. 1).

The Aurora kinases are importantly involved in cell cycle and they exhibit most of their known functions in mitosis. They are involved in some checkpoint regulation pathways including spindle assembly checkpoint, alignment of metaphase chromosomes and chromosomal biorientation. Aberrant expression of Aurora kinases may disturb checkpoint functions particularly in mitosis and this may lead to genetic instability and trigger the development of tumors. Aurora kinases have gained much attention since they were identified as bona fide oncogenes.

\section{Structure}

Aurora kinases, divided into A, B, and $\mathrm{C}$ have an amino acid sequence length ranging from 309-403 (ref. ${ }^{1}$ ). They have N-terminal domain (39-129), a protein kinase domain and C-terminal domain (15-20) (Fig. 2). Aurora $\mathrm{A}$ and $\mathrm{B}$ share $71 \%$ identity in their C-terminal catalytic domain ${ }^{2}$. The high percentage of conservation is very important in relation to the specificity of substrates and inhibitors. The mean proportion of similar amino acids estimated by pair- wise sequence comparisons is significantly higher among different families of Aurora A, B and $C$ in vertebrates $(0.84 \pm 0.5)$ than within the same family (Aurora A or B) in vertebrates and invertebrates species $(0.69 \pm 0.3$ for both). This suggests a recent evolutionary radiation of Aurora families within vertebrates ${ }^{3}$. Structural and motif based comparison suggested an early divergence of Aurora A from Aurora B and Aurora C (ref. ${ }^{4}$ ). Aurora A, B, \&C have been mapped on chromosomes 20q13.2, $17 \mathrm{p} 13.1$, and $10 \mathrm{q} 13$ respectively ${ }^{5-7}$.

Aurora kinases show little variability in their amino acid sequence and this is very important for interaction with different substrates specific for each Aurora kinase and for their different subcellular localizations ${ }^{8}$. ATP binding active site in all Aurora kinases are lined by 26 residues and three variants: Leu215, Thr217, R220 are specific to Aurora A. Aurora A, B, and C share identical sequences in their active site $^{3}$.

\section{Aurora Kinase Geography and Substrates}

Aurora kinases are nuclear proteins having different sub cellular locations. Aurora A localizes within the centrosomes from the time of duplication of centromeres until mitotic exit ${ }^{2}$. Bischoff et al. ${ }^{5}$ using indirect immunofluorescence with Aurora A antibodies found the specific localization of Aurora A. Aurora A localizes to centrosomes, spindle poles, spindle from prophase to metaphase but predominantly to the spindle during telophase ${ }^{5}$ (Fig. 3). Northern blot analysis has confirmed Aurora A expression in thymus, testis and fetal liver and low expression in bone marrow, lymph node and spleen ${ }^{5}$. Late mitosis or early G1 stage is the degradation point for Aurora A. Aurora B, known as chromosomal passenger protein localizes to kinetochores from prophase to metaphase, in the mid zone during anaphase and eventually to the midbody in cytokinesis $^{5}$ (Fig. 3). Northern blot has shown that human Aurora B expression level is high in normal thymus and fetal liver ${ }^{5}$. Aurora $\mathrm{C}$ localizes to centrosomes from anaphase to cytokinesis ${ }^{9}$. It is predominantly expressed in testis and isolated from cDNA library?

A number of substrates of Aurora kinases have been reported by several studies. Some substrates activate Aurora kinases, while others are activated by Aurora kinases. The best-known substrate of Aurora A is TPX2. During mitosis Aurora A is activated by autophosphorylation through the interaction with TPX2. Hence TPX2 is considered a substrate and activator of Aurora A (ref. $\left.{ }^{10}\right)$. Other substrates of Aurora A include LIM protein ${ }^{11}$, Eg5 (ref. ${ }^{12}$ ), CDC25B (ref. ${ }^{13}$ ), p53 (ref. ${ }^{14}$ ), and BRCA-1 (ref. ${ }^{15}$ ). Substrates for Aurora B include histone H3 (Ser 10) (ref. ${ }^{16}$ ), MCAK (ref. ${ }^{17}$ ), histone H2A (T119) $\left(\right.$ ref. $\left.^{18}\right)$, Topoisomerase II $\left(\right.$ ref. $\left.^{19}\right)$, INCENP, survivin ${ }^{20}$, and CENP-A (ref. ${ }^{21}$ ). The only best-known substrate for Aurora C is INCENP $\left(\right.$ ref. $^{22}$ ). Identification of further substrates is important to develop biomarkers for assessing the efficacy of targeted drug cancer treatment.

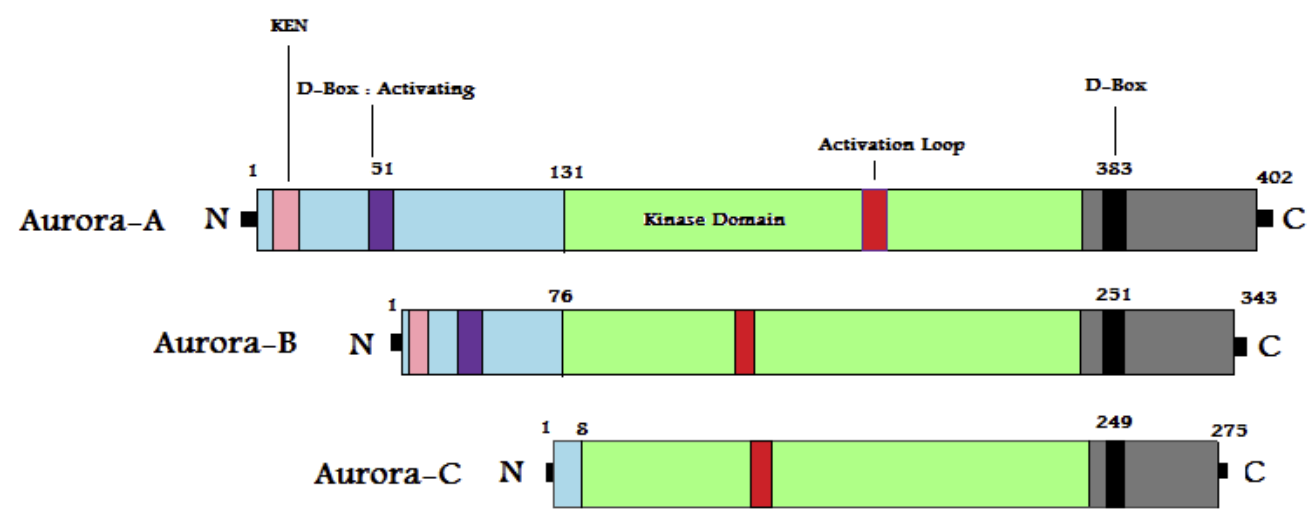

Fig. 2. Schematic diagram representing the Aurora A, B, \& C kinases domains. N \& $C$ terminal domains contain most of the regulatory sequences. The central domain consist of catalytic kinase domain and activation loop. D-Box at the c-terminal domain is the destruction box. 
Prophase Metaphase Telophase

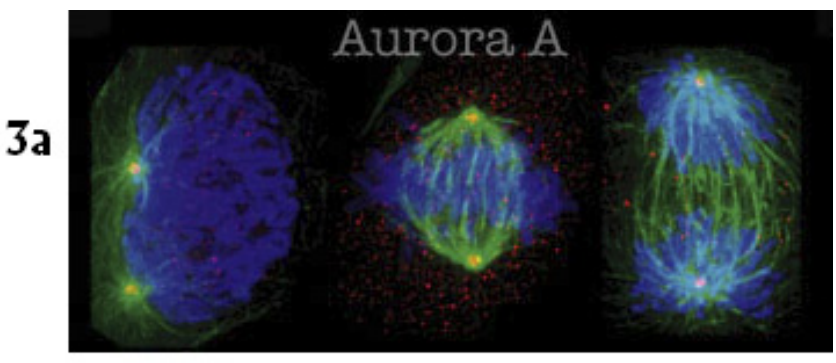

3b

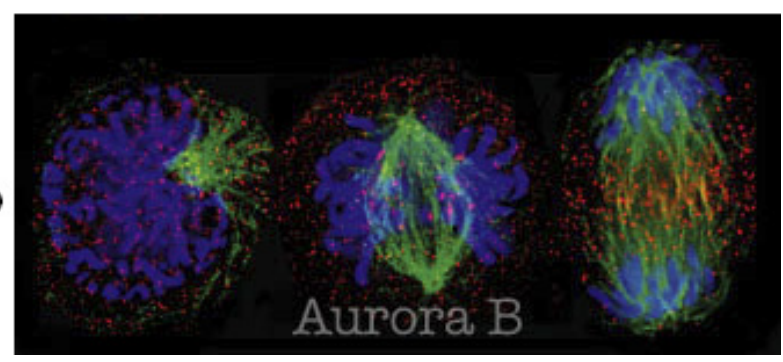

Prophase
Metaphase

Telophase

Fig. 3. Comparative localization of Aurora A and B. (a) Aurora A in prophase predominantly stained (green) around centrosomes, on the microtubules near spindle poles in metaphase and polar microtubules during anaphase \& telophase. (b) Aurora B (red) localized to inner centromere during prophase and metaphase, spindle mid-zone microtubules in anaphase, and subsequently localized to midbody during cytokinesis. (Images taken from the permission of Dr. Andrews, P.D. Home page, University of Dundee)

\section{Aurora Kinases Functions}

Aurora kinases are involved in multiple functions of mitosis. Aurora A is involved in mitotic entry, separation of centriole pairs, accurate bipolar spindle assembly, and alignment of metaphase chromosomes and completion of cytokinesis $^{23}$. The activity of Aurora A is closely related to centrosomes. It plays a role in bipolar spindle assembly, maturation of duplicated centrosomes by recruiting proteins including D-Tacc ${ }^{24}, \gamma$-tubulin ${ }^{25}$, SPD-2 $\left(\right.$ ref. $\left.^{26}\right)$, and centromeric $\mathrm{ChToh}^{27}$. Recently role of Aurora A in the promotion of nuclear envelop breakdown has been described $^{28}$. Aurora B is one of the main components of the chromosomal passenger complex, which is a functional mitotic structure. It is involved in chromosomal bi-orientation, regulating kinetochores microtubule association and cytokinesis ${ }^{29}$. Incorrect attachments of sister chromatid kinetochores to microtubules can be resolved by Aurora $B^{17,30}$ (Fig. 4). Inhibition of Aurora B by small molecule inhibitor, Hesperadin, significantly increased syntelic attachment, which may lead to genetic instability $^{30}$ (Fig. 4). Aurora B is specifically enriched at merotelic attachment sites and is involved in the release of

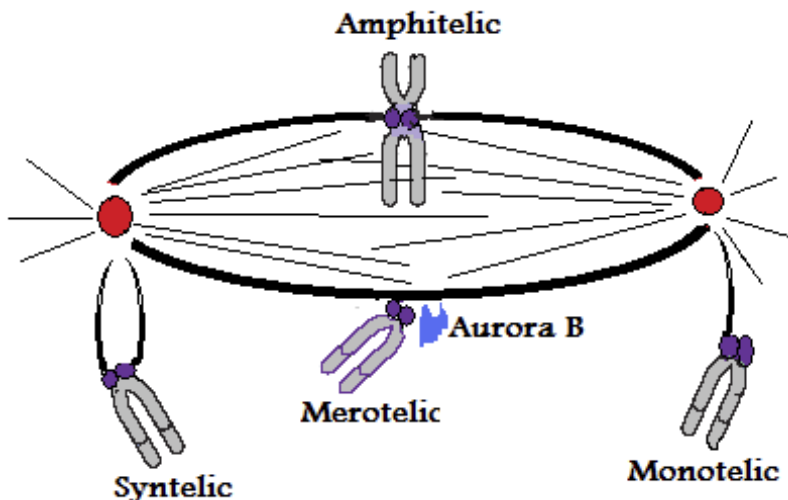

Fig. 4. Schematic diagram of centrosomes (red), spindle (microtubule-black lines), various chromosome orientations (gray) or attachments to spindle microtubules, and Aurora B (blue). Amphitelic attachment of sister chromatid kinetochores, where both the kinetochores are attached to opposite poles is the correct attachment (biorientation). Defects in chromosome biorientation leads to monotelic (one kinetochore attached to spindle pole), merotelic (one kinetochore attached to both spindle poles), and Syntelic attachments (both kinetochores attached to same spindle pole). Aurora B is involved in correcting merotelic and syntelic attachments. Aberrant expression of Aurora B compromises chromosomal biorientation (amphitelic) leading to genetic instability (aneuploidy).

improper kinetochore microtubule attachments during chromosomal bi-orientation ${ }^{17}$ (Fig. 4). Aurora B phosphorylates histone $\mathrm{H} 3$ (Ser 10), which is believed to aid in chromatin condensation and separation ${ }^{16}$. Aurora $\mathrm{C}$ exhibits similar functions to those assigned to Aurora B and is required for cytokinesis ${ }^{22}$. Hence Aurora $\mathrm{C}$ may be as important a kinase as other kinases in the regulation of various mitotic events.

Recently, the involvement of Aurora B in phosphorylation of centromere-specific histone 2A at T119, which may be crucial for regulation of chromatin structure and function, has been described ${ }^{18}$. Li et al..$^{22}$ found that direct association with INCENP activates Aurora C, suggesting the cooperation of Aurora A and B in the regulation of mitotic events. This led to the question, whether Aurora C can fulfill the fucntions of Aurora B. Indeed, recent publications show that Aurora $\mathrm{C}$ can complement the functions of Aurora B. It co-localizes with Aurora B and survivin as a chromosomal passenger protein and is able to rescue polyploidy induced by inactive Aurora B. In summary Aurora $\mathrm{C}$ appears to exhibit functions similar to those for Aurora B and is required for cytokinesis ${ }^{22}$. 


\section{Regulation of Aurora Kinases}

Phosphorylation, dephosphorylation are the two predominant mechanisms regulating Aurora A activity most of which have been deduced from invertebrates. Generally, phosphorylation of Aurora kinase stimulates kinase activity and three phosphorylation sites in Xenopus have been identified by mass spectrometry. Littlepage, L.E. et al. mapped recombinant Aurora A by mass spec sequencing activated by metaphase arrested Xenopus eggs extracts ${ }^{31}$. Three phosphorylation sites Ser-53, Thr-295, and Ser-349 were identified. Mutations in Thr-295 and Ser-349 reduced or abolished the activity of Aurora $\mathrm{A}^{31}$. Specifically phosphrylation of Thr-295 is required for kinase activation and a protein kinase (PKA) can phosphorylate (Thr-295) and activate the kinase In vitro ${ }^{31}$. Mutation in Ser-53 abolished Cdh1 mediated degradation, showing that phosphorylation is invovled in the regualtion of degradation. PP1 phosphatase negatively regulates Aurora A by dephosphorylating T288 (ref. ${ }^{32}$ ). In mitotsis Aurora A is activated by autophosphorylation through the interaction with TPX2 and could be at least partly due to PP1 antogonism ${ }^{10}$. Kei Honda et al. ${ }^{33}$ demonstrated that Aurora A is turned over by APC-ubiquitin-proteasome pathway. Aurora A degradation is mediated by Cdh1 or Fizzy related once the D-box is recognized ${ }^{34}$.

It has also been shown that Chk1 can phosphorylate Aurora B In vitro, enhancing the kinase activity to augment spindle checkpoint signalling ${ }^{35}$. To date the best known regulators of Aurora B include Survivin and INCENP. Survivin binds to the catalytic domain of Aurora B and enhances the kinase activity and targeting to its substrates ${ }^{36}$. Lower levels of phosphorylated Histone $\mathrm{H} 3$ correlates with the absence of Survivin, confirming that Aurora B activity is enhanced by survivin ${ }^{36}$. Survivin is also involved in the localization of Aurora B to different locations during mitotsis. Kie Honda et al. described activation of Aurora B by C-terminal region of INCENP $\left(\right.$ ref. $\left.{ }^{37}\right)$. Aurora B is activated upon binding of INCENP resulting in the phosphorylation of the conserved motif of INCENP. These events are critical for full activation of Aurora B/INCENP complex ${ }^{37}$. CENP-A is also invlolved in the regualtion of Aurora B localization to centromeres and spindle midzone ${ }^{21}$. Cdh1 and cdc20 are involved in Aurora B degradation and require intact KEN boxes and A-boxes, which are located within the first 65 amino acids ${ }^{38}$. Levels of Aurora $C$ peak later stages of mitosis after Aurora B. Aurora C is regulated by INCENP like Aurora $\mathrm{B}$, through the $\mathrm{C}$-terminal region. Aurora $\mathrm{C}$ may rescue the genetic stability of the cells complementing Aurora B functions in its absence ${ }^{39}$. However regulation of Aurora $\mathrm{C}$ is not completely known and further work is needed. Aurora $\mathrm{C}$ is predicted to turn over by APC-ubiquitin-proteasome pathway through the recognition of D-box.

\section{Aurora Kinases and Cancer}

Aurora kinases perform important functions during mitosis and hence their aberrant expression can lead to the cell transformations underlying cancer. In many tissues, Aurora kinase over-expression leads to genetic instability (aneuploidy), which may cause cancer. Aneuploidy, a condition where the cells have altered, DNA content may arise from mitotic defects including centrosome duplication, centrosome separation, cytokinesis and chromosomal bi-orientation errors. In all these processes Aurora kinases are involved. Therefore it is tempting to state that the aberrant expression of Aurora kinases may lead to aneuploidy. Hence Aurora genes have been classified as bona fide oncogenes.

Aurora A gene was first named as BTAK (Breast Tumor Activated Kinase), because its mRNA is found to over-expressed in breast tumors and plays a critical role in breast tumor cells transformation ${ }^{40}$. In fact the

Table 1. Over-expression or amplification of Aurora kinases in wide variety of tumors types, making them as an attractive targets.

\begin{tabular}{|c|l|c|}
\hline Aurora Kinase & \multicolumn{1}{|c|}{ Tumor type } & Reference \\
\hline Aurora A & Breast Cancer & {$[41]$} \\
Aurora A & Human Gliomas & {$[48]$} \\
Aurora A & Ovarian & {$[41]$} \\
Aurora A & Prostrate & {$[41,49]$} \\
Aurora A & Cervical & {$[41]$} \\
Aurora A & Colon & {$[41]$} \\
Aurora A & Pancreatic & {$[50]$} \\
Aurora A & Lung Cancer & {$[43]$} \\
& & \\
Aurora B & Colon Cancer & {$[44]$} \\
Aurora B & Thyroid cancer & {$[45]$} \\
Aurora B & Oral & {$[46]$} \\
Aurora B & Non small cell lung carcinoma & {$[47]$} \\
Aurora B & Breast cancer & {$[42]$} \\
Aurora C & & \\
& Breast cancer & {$[9]$} \\
\hline
\end{tabular}


presence of $20 \mathrm{q} 13$ amplicon in breast tumor is a poor prognostic indicator. Two groups independently reported that ectopic over-expression of Aurora A can transform NIHT3 and rat 1 cells ${ }^{5}$, which can induce tumors in nude mice. These reports are a break-through and the Aurora kinases came into light attracting more attention than ever before. Zhou et al. ${ }^{41}$ performed northern-blot and southern-blot and reported 2.5-8 fold amplification of Aurora A in many cell tumors including breast (BT474, MDA-MB-231), ovarian (2774, SK-OV3), colon (HCT116, HT29, SW480), prostate (DU145, PC3), leukemia (HL60, K562), neural (HTB10) and cervical (SW756). Functional polymorphism due to transitions resulting Phe31Ile has been observed and polymorphism is associated strongly with human colon tumors ${ }^{42}$. Recently similar polymorphisms have been reported in lung cancer predominant in Caucasians ${ }^{43}$. Apart from these tumor types, Aurora A over-expression has been reported in many other tumors (Table 1). Taken together these data, suggest that Aurora A can be characterized as a bona fide oncogene.

Aberrant expression of Aurora B also induces tumor formation and this is not surprising as the kinase is involved in many functions during mitosis. Hence it is logical to classify Aurora B as an oncogene. Katayama et al. performed in situ hybridization, northern and western blot studies from surgically resected colon tumor specimens and reported the over-expression of Aurora B and tumor progression ${ }^{44}$. This significant research stimulated interest in the development of new drugs against Aurora B. Malignant progression of thyroid anaplastic carcinoma cells correlates with the over-expression of Aurora $\mathrm{B}^{45}$. Over-expression of Aurora B has also been reported in oral cancer ${ }^{46}$ and primary non-small cell lung carcinoma ${ }^{47}$. Aurora B has found to be over-expressed in some other tumor types (Table 1). Aurora $\mathrm{C}$ has been found to be over-expressed in some cancer cell lines including HepG2, HUH7, MDA-MB-453 and HeLa. However its correlation with cancer progression is unclear ${ }^{9}$. Hence active research is underway to determine Aurora $\mathrm{C}$ as an oncogene.

Based on these reports it is reasonable to classify Aurora kinases as oncogenes. They are not aberrantly expressed in one particular type of tumors only, unlike some other oncogenic kinases. Hence developing potential Aurora kinase inhibitors can target wide range of tumor types. Development of drugs that focus on the Aurora kinases could be promising in the treatment of various cancers. Some Aurora kinases inhibitors have been developed recently and indeed some drugs are in preclinical stage and phase 1 and phase 11 clinical trials. Further research on Aurora kinases is required to determine their regulation and their interaction with other partners.

\section{CONCLUSION}

Chromosomal duplication and cell division are very critical, where one copy of each duplicated chromosome segregates to each of two daughter cells. In order to ensure the fidelity of the cell cycle, various proteins regulate the orderly events at each stage of the cell cycle checkpoints. Mutations or aberrant expression of these proteins involved in the cell cycle regulation may lead to tumorigenesis. The importance of evolutionary conserved Aurora kinases in the regulation of mitotic events came into light recently. They are involved in the regulation of microtubule dynamics, chromosomal segregation, and cytokinesis. These kinases are frequently over-expressed in human tumors resulting in genomic instability. Hence they are clearly implicated in tumorigenesis. Recently Aurora kinases are considered as interesting targets for the development of anticancer drugs. Some Aurora kinases developed recently have proven effective in the clinic on a wide range of tumors. Further understanding the regulation and functions of Aurora kinases deeply, may help to develop potentially useful drugs for targeted cancer treatment.

\section{ACKNOWLEDGEMENTS}

This work was kindly supported in parts by grants awarded by the Czech Ministry of Education (MSM 6198959216 and LC07017). We are thankful to Dr. Andrews, P.D. University of Dundee for kindly providing Aurora $A$ and Aurora B immunohistochemical images.

\section{REFERENCES}

1. Bolanos-Garcia M. Aurora Kinases. Int. J. Biochem. \& Cell Biol. 2004; 37:1572-7.

2. Carmena M, Earnshaw WC. The Cellular Geography of Aurora Kinases. Nat. Rev. Mol. Cell. Biol. 2003; 4:842-54.

3. Brown JR, Koretke KK, Birkeland ML, Sanseau P, Patrick DR. Evolutionary relationships of Aurora kinases: Implications for model organism studies and the development of anti-cancer drugs. BMC Evol. Biol. 2004; 4:1-10.

4. Cheetam GMT, Knegtel RMA, Coll JT, Renwick SB, Swenson L, Weber P, Lippke, JA, Austen DA. Crystal Structure of Aurora2, an Oncogenic Serine/Threonine Kinase. J. Biol. Chem. 2000; 277:42419-22.

5. Bischoff JR, Anderson L, Zhu Y, Mossie K, Ng L, Souza B, Schryver B, Flanagan P, Clairvoyant F, Ginther C, Chan CS, Novotny M, Slamon DJ, Plowman GD. A Homologue of Drosophila Aurora kinase is Oncogenic and amplified in human colorectal cancers. EMBO J. 1998; 17:3052-65.

6. Kimura M, Matsuda Y, Yoshioka T, Sumi N, Okano Y. Identification and characterization of STK12/Aik2: a human gene related to aurora of Drosophila and yeast IPL1. Cytogenet. Cell Genet. 1998; 82:147-52.

7. Bernard M, Sanseau P, Henry C, Couturier A, Prigent C. Cloning of STK13, a third human protein kinase related to Drosophila aurora and budding yeast Ipl1 that maps on chromosome 19q13.3-ter. Genomics 1998; 53:406-9.

8. Adams RR. Carmena M, Earnshaw WC. Chromosomal passengers and the (aurora) ABCs of mitosis. Trends Cell Biol. 2001; 1:49. 54

9. Kimura M, Matsuda Y, Yoshioka T, Okano Y. Cell cycle-dependent expression and centrosome localization of a third human Aurora/ Ipl1-related protein kinase, AIK3. J. Biochem. 1999; 274:733440

10. Eyers PA, Erikson E, Chen LG, Maller JL. A novel mechanism for activation of the protein kinase Aurora A. Curr. Biol. 2003; 13:691-7. 
11. Hirota T, Kunitoku N, Sasayama T, Marumoto T, Zhang D, Nitta M, Hatakeyama K, Saya H. Aurora-A and an interacting activator, the LIM protein Ajuba, are required for mitotic commitment in human cells. Cell 2003; 114:585-98.

12. Giet R, Uzbekov R, Cubizolles F, Le Guellec K, Prigent C. The Xenopus laevis aurora-related protein kinase $\mathrm{pEg} 2$ associates with and phosphorylates the kinesin-related protein XIEg5. J. Biol. Chem. 1999; 274:15005- 13.

13. Dutertre S, Cazales M, Quaranta M, Froment C, Trabut V, Dozier C, Mirey G, Bouché JP, Theis-Febvre N, Schmitt E, Monsarrat B, Prigent C, Ducommun B. Phosphorylation of CDC25B by AuroraA at the centrosome contributes to the G2-M transition. J. Cell. Sci. 2004; 117:2523-31.

14. Liu Q, Kaneko S, Yang L, Feldman RI, Nicosia SV, Chen J, Cheng JQ. Aurora-A Abrogation of p53 DNA binding and transactivation activity by phosphorylation of serine 215. J. Biol. Chem. 2004; 279:52175-82.

15. Ouchi M, Fujiuchi N, Sasai K, Katayama H, Minamishima YA, Ongusaha PP, Deng C, Sen S, Lee SW, Ouchi T. BRCA1 phosphorylation by Aurora-A in the regulation of $\mathrm{G} 2$ to $\mathrm{M}$ transition. J. Biol. Chem. 2004; 279:19643-8.

16. Goto H, Yasui Y, Nigg EA, Inagaki M. Aurora B phosphorylates Histone $\mathrm{H} 3$ at serine 28 with regard to the mitotic chromosome condensation. Genes Cells 2002; 7:11-7.

17. Knowlton AL, Lan W, Stukenberg P. Aurora B is enriched at merotelic attachment sites, where it regulated MCAK. Curr. Biol. 2006; 16:1705-10.

18. Brittle AL, Nanba Y, Ito T, Ohkura H. Concerted action of Aurora B, Polo and NHK-1 kinases in centromere-specific histone 2A phosphorylation. Exp. Cell Res. 2007; 313:2780-5.

19. Morrison C, Henzing AJ, Jensen ON, Osheroff N, Dodson H, Kandels-Lewis SE, Adams RR, Earnshaw WC. Proteomic analysis of human metaphase chromosomes reveals topoisomerase II alpha as an Aurora B substrate. Nucleic Acids Res. 2002; 30:5318-27.

20. Speliotes EK, Uren A, Vaux D, Horvitz HR. The survivin-like C. elegans BIR-1 protein acts with the Aurora-like kinase AIR-2 to affect chromosomes and the spindle midzone. Mol. Cell 2000; 6:211- 23.

21. Zeitlin SG, Shelby RD, Sullivan KF. CENP-A is phosphorylated by Aurora B kinase and plays an unexpected role in completion of cytokinesis. J. Cell Biol. 2001; 155:1147-57.

22. Li X, Sakashita G, Matsuzaki H, Sugimoto K, Kimura K, Hanaoka F, Taniguchi H, Furukawa K, Urano T. Direct association with inner centromere protein (INCENP) activates the novel chromosomal passenger protein, Aurora-C. J. Biol. Chem. 2004; 279:47201-11.

23. Marumoto T, Honda S, Hara T, Nitta M, Hirota T, Kohmura E, Saya H. Aurora-A Kinase Maintains the Fidelity of Early and Late Mitotic events in HeLa Cells. J. Biol. Chem. 2003; 278:51786-95.

24. Berdnik D, Knoblich, JA. Drosophila Aurora-A is required for centrosome maturation and actin-dependent asymmetric protein localization during mitosis. Curr. Biol. 2002; 12:640-7.

25. Hannak E, Kirkham M, Hyman AA, Oegema K. Aurora-A kinase is required for centrosome maturation in Caenorhabditis elegans. J. Cell Biol. 2001; 155:1109-16.

26. Kemp CA, Kopish KR, Zipperlen P, Ahringer J, O'Connell KF. Centrosome maturation and duplication in $\mathrm{C}$. elegans require the coiled-coil protein SPD-2. Dev. Cell 2004; 6:511-23.

27. Conte N, Delaval B, Ginestier C, Ferrand A, Isnardon D, Larroque C, Prigent C, Séraphin B, Jacquemier J, Birnbaum D. TACC1-chTOG-Aurora A protein complex in breast cancer. Oncogene 2003; 22:8102-16.

28. Portier N, Audhya A, Maddox PS, Green RA, Dammermann A, Desai A, Oegema K. A microtubule-independent role for centrosomes and Aurora A in nuclear envelope breakdown. Dev. Cell 2007; 12:515-29.

29. Adams RR, Maiato H, Earnshaw WC, Carmena M. Essential Roles of Drosophila Inner Centromere Protein (INCENP) and Aurora B in Histone H3 Phosphorylation, Metaphase Chromosome Alignment, Kinetochore Disjunction, and Chromosome Segregation. J. Biol. Chem. 2001; 15:865-80.
30. Hauf S, Cole RW, LaTerra S, Zimmer C, Schnapp G, Walter R, Heckel A, van Meel J, Rieder CL, Peters JM. The small molecule Hesperadin reveals a role for Aurora B in correcting kinetochoremicrotubule attachment and in maintaining the spindle assembly checkpoint. J.Biol.Chem. 2003; 16:281-94.

31. Littlepage LE, Wu H, Andresson T, Deanehan JK, Amundadottir LT, Ruderman JV. Identification of phosphorylated residues that affect the activity of the mitotic kinase Aurora A. Proc. Natl. Acad. Sci. U.S.A. 2002; 99:15440-5.

32. Walter AO, Seghezzi W, Korver W, Sheung J, Lees E. The mitotic serine/threonine kinase Aurora $2 / \mathrm{AIK}$ is regulated by phosphorylation and degradation. Oncogene 2000; 19:4906-16.

33. Honda K, Mihara H, Kato Y, Yamaguchi A, Tanaka H, Yasuda H, Furukawa K, Urano T. Degradation of human Aurora2 protein kinase by the anaphase-promoting complex-ubiquitin-proteasome pathway. Oncogene 2000; 19:2812-9.

34. Castro A, Arlot-Bonnemains Y, Vigneron S, Jean-Claude L, Prigent C, Lorca T. APC/Fizzy-Related targets Aurora-A kinase for proteolysis. EMBO Rep. 2002; 3:457-62.

35. Zachos G, Black E, Walker M, Scott M, Vagnarelli P, Earnshaw W. Chk1 is required for spindle checkpoint fucntion. Dev. Cell 2007; 12:247-60.

36. Chen J, Jin S, Tahir SK, Zhang H, Liu X, Sarthy AV, McGonigal TP, Liu Z, Rosenberg SH, Ng SC. Survivin enhances Aurora-B kinase activity and localizes Aurora B in human cells. J. Biochem. 2003; 278:486-90.

37. Honda R, Korner R, Nigg EA. Exploring the functional interactions between Aurora B, INCENP, and survivin in mitosis. Mol. Biol. Cell 2003; 14:3325-41.

38. Nguyen HG, Chinnappan D, Urano T, Ravid K. Mechanism of Aurora-B Degradation and Its Dependency on Intact KEN and ABoxes: Identification of an Aneuploidy-promoting Property. Mol. Cell. Biol. 2005; 25:4977-92.

39. Sasai K, Katayama H, Stenoien DL, Fujii S, Honda R, Kimura M, Okano Y, Tatsuka M, Suzuki F, Nigg EA, Earnshaw WC, Brinkley WR, Sen S. Aurora-C is a novel chromosomal passenger protein that can complement Aurora-B kinase function in mitotic cells. Cell Motil. Cytoskeleton 2004; 59:249-63.

40. Sen S, Zhou H, White RA. A putative serine/threonine kinase encoding gene BTAK on chromosome $20 \mathrm{q} 13$ is amplified and overexpressed in human breast cancer cell lines. Oncogene 1997; 14:2195-200.

41. Zhou H, Kuang J, Zhong L, Kuo WL, Gray JW, Sahin A, Brinkley BR, Sen S. Tumour amplified kinase STK15/BTAK induces centrosome amplification, aneuploidy and transformation. Nat. Genet. 1998; 20:189-93.

42. Tchatchou S, Wirtenberger M, Hemminki K, Sutter C, Meindl A, Wappenschmidt B, Kiechle M, Bugert P, Schmutzler RK, Bartram CR, Burwinkel B. Aurora kinases A and B and familial breast cancer risk. Cancer Lett. 2007; 247:266-72.

43. Gu J, Gong Y, Huang M, Lu C, Spitz MR, Wu X. Polymorphisms of STK15 (Aurora-A) gene and lung cancer risk in Caucasians. Carcinogenesis 2007; 28:350-5.

44. Katayama H, Ota T, Jisaki F, Ueda Y, Tanaka T, Odashima S, Suzuki F, Terada Y, Tatsuka M. Mitotic kinase expression and colorectal cancer progression. J. Natl. Cancer Inst. 1999; 91: 1160-2.

45. Sorrentino R, Libertini S, Pallante PL, Troncone G, Palombini L, Bavetsias V, Spalletti-cernia D, Laccetti P, Linardopoulos S, Chieffi P, Fusco A, Portell G. Aurora B overexpression associates with the thyroid carcinoma undifferentiated phenotype and is required for thyroid carcinoma cell proliferation. J. Clin. Endocrinol. Metab. 2004; 90:928-35.

46. Guangying Q, Ikuko O, Yasusei K, Mutsumi M, Siriwardena B, Fumio S, Masaaki M, Takashi A. Aurora-B overexpression and its correlation with cell proliferation and metastasis in oral cancer. Springer 2007; 450:297-302.

47. Smith SL, Bowers NL, Betticher DC, Gautschi O, Ratschiller D, Hoban PR, Booton R, Santibáńez-Koref MF, Heighway J. Overexpression of aurora $\mathrm{B}$ kinase (AURKB) in primary non-small 
cell lung carcinoma is frequent, generally driven from one allele, and correlates with the level of genetic instability. Br. J. of cancer 2005; 93:719-29.

48. Reichardt W, Jung V, Brunner C, Klein A, Wemmert S, Romeike BFM, Zang KD, Urbschat S. The putative serine/threonine kinase gene STK15 on chromosome 20q13.2 is amplified in human gliomas. Oncol. Rep. 2003; 10:1275-9.
49. Buschhorn HM, Klein RR, Chambers SM, Hardy MC, Green S, Bearss D, Nagle RB. Aurora-A over-expression in high-grade PIN lesions and prostate cancer. The Prostrate 2005; 64:341-6.

50. Li D, Zhu J, Firozi PF, Abbruzzese JL, Evans DB, Cleary K, Friess $\mathrm{H}$, Sen S. Overexpression of oncogenic STK15/BTAK/Aurora A kinase in human pancreatic cancer. Clin. Cancer Res. 2003; 9:991-7. 